\title{
Pansharpening based on convolutional autoencoder and multi-scale guided filter
}

Ahmad AL Smadi ${ }^{1}$, Shuyuan Yang ${ }^{1 *}$, Zhang Kai $^{2}$, Atif Mehmood ${ }^{1}$, Min Wang $^{3}$ and Ala Alsanabani ${ }^{1}$

*Correspondence: syyang2009@gmail.com

'School of Artificial Intelligence, Xidian University, No. 2 South Taibai Road, 710071 Xian, China Full list of author information is available at the end of the article

\begin{abstract}
In this paper, we propose a pansharpening method based on a convolutional autoencoder. The convolutional autoencoder is a sort of convolutional neural network (CNN) and objective to scale down the input dimension and typify image features with high exactness. First, the autoencoder network is trained to reduce the difference between the degraded panchromatic image patches and reconstruction output original panchromatic image patches. The intensity component, which is developed by adaptive intensity-hue-saturation (AlHS), is then delivered into the trained convolutional autoencoder network to generate an enhanced intensity component of the multi-spectral image. The pansharpening is accomplished by improving the panchromatic image from the enhanced intensity component using a multi-scale guided filter; then, the semantic detail is injected into the upsampled multi-spectral image. Real and degraded datasets are utilized for the experiments, which exhibit that the proposed technique has the ability to preserve the high spatial details and high spectral characteristics simultaneously. Furthermore, experimental results demonstrated that the proposed study performs state-of-the-art results in terms of subjective and objective assessments on remote sensing data.
\end{abstract}

Keywords: Pansharpening, Convolutional autoencoder, Guided image filtering, Adaptive intensity-hue-saturation AlHS

\section{Introduction}

There are many applications based on remote sensing satellites that require observation of the alterations of the earth, such as image fusion [1-3] and mapping land cover [4]. Given that, pansharpening is one of the essential interests of many scientists. It is difficult that the remote sensing satellites can obtain a panchromatic image (PAN) and a multi-spectral image (MS) with the qualities of both high spatial resolution and high spectral resolution at the same time due to data transmission impediment. However, the main objective of pansharpening is fusing the high spatial resolution PAN image with the corresponding high spectral resolution MS image to acquire high spatial and spectral resolutions for MS image [5].

As indicated by [6-8], a wide assortment of image fusion techniques can be classified into two classes based on the way of extracting a spatial detail from a PAN image: (1) component substitution (CS) and (2) multi-resolution analysis (MRA). And some methods do

(c) The Author(s). 2021 Open Access This article is licensed under a Creative Commons Attribution 4.0 International License which permits use, sharing, adaptation, distribution and reproduction in any medium or format, as long as you give appropriate credit to the original author(s) and the source, provide a link to the Creative Commons licence, and indicate if changes were made. The images or other third party material in this article are included in the article's Creative Commons licence, unless indicated otherwise in a credit line to the material. If material is not included in the article's Creative Commons licence and your intended use is not permitted by statutory regulation or exceeds the permitted use, you will need to obtain permission directly from the copyright holder. To view a copy of this licence, visit http://creativecommons.org/licenses/by/4.0/. 
not belong to these two categories, such as model-based pansharpening method $[9,10]$. Among the conventional component substitution-based methods include intensity-huesaturation (IHS) [11], principal component analysis (PCA) [12], Gram-Schimidt [13], and Brovey transform [14], etc. in which the detail information is extracted by the difference between the PAN image and linear combination of the upsampled MS image; therefore, the component substitution-based methods have a spectral distortion in the fused image. In contrast, the multi-resolution analysis-based methods, such as Smoothing Filter-based Intensity Modulation (SFIM) [15], generalized Laplacian pyramid (MTF-GLP) [16], and indusion [17], extract the detail information by the difference between the PAN image and its low resolution. These methods offer an outstanding spectral resolution, but they suffer from spatial distortion in the fused image. The edge-preserving filtering techniques have drawn an important role in pansharpening. Guided image filter [18] is one of the wellknown techniques. Yang et al. [19] introduced multi-scale guided filer based on adaptive intensity-hue-saturation (MSGF); they used the intensity image as a guidance image to enhance the PAN image. In our work, the multi-scale guided filter is used to enhance the semantic detail map by utilizing the enhanced intensity image as a guidance image that is obtained by CAE.

Recently, the use of deep neural networks has been a hot topic in many fields [2025]. Researchers have started investigating this topic for pansharpening. Scarpa et al. [21] proposed the convolutional neural network-based pansharpening method.

Residual convolutional neural network ( $\mathrm{RCNN}$ ) was utilized to achieve pansharpening [26]. Huang et al. [27] introduced a pansharpening model using deep neural networks $(\mathrm{DNN})$, which utilized the relationship between PAN image patches and MS image patches for training the neural network. More recently, in [28], convolutional autoencoder (CAE)-based multi-spectral image fusion was introduced in which the low-resolution MS images is fed into the trained CAE to generate estimated high-resolution MS images; then, the fusion process is achieved by injecting the detailed map of each image into the corresponding estimated high-resolution MS bands. Inspired by this, we propose a pansharpening technique based on a convolutional autoencoder. First, the convolutional autoencoder is trained from the degraded PAN image patches to generate the original PAN image patches; the AIHS component is then tested on the trained network to obtain enhanced intensity components. Further, the guided filter is employed to enhance the PAN image using the enhanced intensity component. Finally, the experiments are conducted on both real and degraded datasets. We showed that the fusion process of the convolutional autoencoder with a guided filter is capable of preserving the high spatial details and high spectral characteristics simultaneously, which is a start-of-the-art approach on multiple tasks. And our method is also more robust against spectral and spatial distortions.

\subsection{Convolutional autoencoder}

Autoencoder belongs to unsupervised learning that considers an input image and attempts to reconstruct it back. The convolutional autoencoder is a sort of convolutional neural network that reproduces the input image patches at the output. However, the design of a convolutional autoencoder comprises two fundamental phases, which are the encoding phase and the decoding phase. The encoding phase represents half of the network, and it incorporates convolution and max-pooling layers. In contrast, 
the decoding phase for recreating the input image patches from the degraded pieces comprises deconvolution and upscaling layers [29].

\subsubsection{Encoding phase}

A convolution among an input volume $I=\left\{I_{1}, \cdots, I_{D}\right\}$ with $D$ dimension and every convolutional layer is composed of $n$ convolutional filters $F^{(1)}=\left\{F_{1}^{(1)}, \ldots, F_{\mathrm{n}}^{(1)}\right\}$ which is considered to produce $m$ features.

$$
O_{m}=a\left(I * F_{m}^{(1)}+b_{m}^{(1)}\right) \quad m=1,2, \cdots, n
$$

$O_{m}$ represents the feature maps of the input $I, b_{m}$ represents the bias, and $a$ denotes an activation function.

\subsubsection{Decoding phase}

The produced $m$ feature maps considered to be used as input to the decoder, to reconstruct the input image, which is obtained by the consequence of the convolution between $O=\left\{O_{i=1}\right\}^{n}$ with convolutional filters $F^{(2)}=\left\{F_{1}^{(2)}, \ldots, F_{\mathrm{n}}^{(2)}\right\}$ that estimated as follows:

$$
\tilde{I}=a\left(O * F_{\mathrm{m}}^{(2)}+b_{\mathrm{m}}^{(2)}\right)
$$

Considering that both the output image patches and its input have the same dimension, therefore, it is conceivable to relate $I$ and $\tilde{I}$ using a loss function to update the weights during training, for example, mean square error (MSE).

$$
\mathcal{L}(I, \tilde{I})=\frac{1}{2}\|I-\tilde{I}\|_{2}^{2}
$$

\subsection{Adaptive intensity-hue-saturation}

The IHS technique belongs to CS-based methods that introduced [30], and it is just appropriate for MS images with three bands [11]. Even though the IHS strategy displays extraordinary spatial quality, it severely experiences spectral distortion. The general formula for generating an intensity component is as follows:

$$
I=\sum_{i=1}^{n} \alpha_{i} M_{i^{\prime} t h}
$$

where $\alpha_{i}$ denotes the weight coefficients, and $n$ represents the number of spectral bands. $M_{i}$ indicates the $i_{t h}$ band of the upsampled MS band. Therefore, Rahmani et al. [31] AIHS was introduced, in which the optimal weights are obtained by solving the following optimization problem:

$$
\alpha_{i}^{*}=\arg \min _{\alpha_{\mathrm{i}}}\left\|P A N-\sum_{\mathrm{i}=1}^{\mathrm{n}} \alpha_{\mathrm{i}} \mathbf{M}_{i^{\prime} t h}\right\|^{2}
$$

where PAN denotes panchromatic image.

\subsection{Guided filter}

The guided filter GF was introduced by He et al. [32]. The uses of guided filter have been widely utilized in image processing fields such as detail enhancement and image fusion. The guided filter can maintain a strategic distance from ringing artifacts. The GF depends on a local linear model that is using the guided image gui to filter the input image inp. Therefore, the output image Out can conserve the essential data of the inp and obtain the 
variation trend of gui at the same time [19]. Mathematically, the guided filter is employed to find a pair of scalar values $a_{i}$ and $b_{i}$ that solves the following problem [33]:

$$
\underset{a_{i}, b_{i}}{\operatorname{argmin}} \frac{1}{n}\left\|\mathbf{i n p}_{i}-\left(a_{i} \mathbf{g u i}_{i}+b_{i}\right)\right\|_{2}^{2}+\zeta\left|a_{i}\right|_{2}^{2}
$$

Here, $n$ denotes to the number of pixels in a squared window $\mathbf{w}$ with size $(2 r+1) \times(2 r+1)$, and $\zeta$ is a small regularization constant that prevents large $a_{i}$.

$$
\begin{aligned}
& a_{i}=\frac{\frac{1}{n}\left(\mathbf{i n p}_{i}-\mathbf{i n p}_{i}\right)^{\mathrm{T}}\left(\mathbf{g u i}_{i}-\mathbf{g u i}_{i}\right)}{\frac{1}{n}\left(\mathbf{g u i}_{i}-\mathbf{g u i}_{i}\right)^{\mathrm{T}}\left(\mathbf{g u i}_{i}-\mathbf{g u i}_{i}\right)+\zeta} \\
& =\frac{\operatorname{cov}\left(\mathbf{i n p}_{i}-\mathbf{i n p}_{i}, \mathbf{g u i}_{i}-\mathbf{g u i}_{i}\right)}{\operatorname{var}\left(\mathbf{g u i}_{i}-\mathbf{g u i}_{i}\right)+\zeta} \\
& b_{i}=\mathbf{i n p}_{i}-a_{i} \overline{g u i}_{i}
\end{aligned}
$$

Here, $\mathbf{i n p}_{i}$ and $\mathbf{g u i}_{i}$ represent the input image mean and the guidance image mean, respectively. Thus, after computing $a_{i} ; b_{i}$ for all windows in the image, the filtering output is computed as follows:

$$
\text { Out }_{i}=\bar{a}_{i} \mathbf{i n p}_{i}+\bar{b}_{i}
$$

The following equation represented the guided filter operation in this paper:

$$
\text { Out }=\mathbf{G F}(\text { gui, inp })
$$

\section{Methodology}

In this paper, we propose a pansharpening technique based on a convolutional autoencoder and CS-based method. First, we highlight the steps for building our technology are:

- Utilize the convolutional autoencoder to enhance to enhance the intensity component which is obtained by AIHS from MS and PAN images. And the spatial resolution enhancement of the degraded PAN image is used the to train the model.

- Generate the intensity component of the MS image by utilizing AIHS-based method, which is then fed to trained convolutional autoencoder considering this as a testing step.

- Utilize the estimated intensity component to enhance the PAN image by using the guided filter.

- The fusion step represents the last phase of the proposed technique. However, it will be explained in detail later.

Figure 1 illustrates the schematic of the proposed method.

\subsection{Enhancing the spatial detail}

To enhance the spatial detail of the intensity component, we utilize the convolutional autoencoder network in which the relationship between PAN image patches and its degraded form is learned. Note that the degraded PAN image is generated using bi-cubic interpolation. The convolutional autoencoder is used to minimize the difference between input image patches and reconstruction output original image patches. Figure 2 illustrates the applied structure of the convolutional autoencoder. 


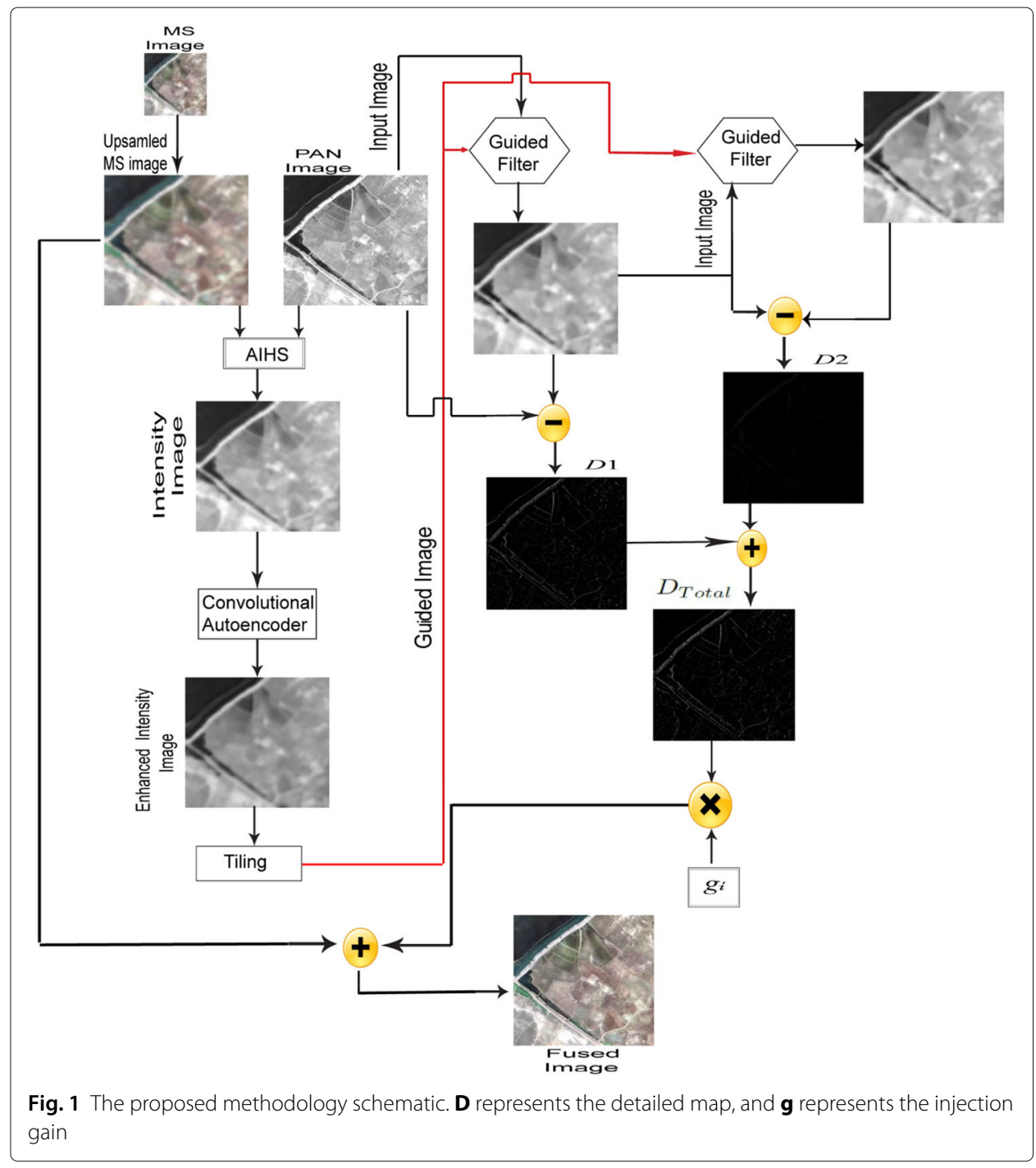

According to [28], the same description of the training network would apply here: the PAN image and its spatially degraded image are partitioned into $8 \times 8$ patches with 5 overlapping pixels that include 500,000 patch pairs, 30 epochs for training, considering that the relationship between PAN image patches and its degraded image patches is learned

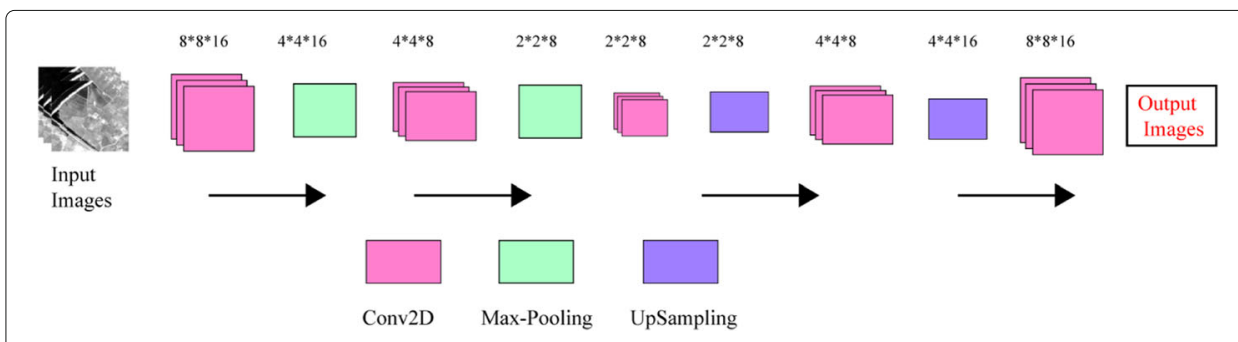

Fig. 2 The structure of the convolutional autoencoder used 
by the training network. The following equation illustrates the output patches of the convolutional autoencoder network at each iteration:

$$
\left\{\tilde{P}_{\mathrm{i}}\right\}_{\mathrm{i}=1}^{\mathrm{n}}=\operatorname{Dec}\left(\operatorname{Enc}\left(\left\{P_{\mathrm{i}}^{\mathrm{L}}\right\}_{\mathrm{i}=1}^{\mathrm{n}}\right)\right)
$$

where $\left\{\tilde{P}_{\mathrm{i}}\right\}_{\mathrm{i}=1}^{\mathrm{n}},\left\{P_{\mathrm{i}}^{\mathrm{L}}\right\}_{\mathrm{i}=1}^{\mathrm{n}}$ represent the output and input patches, respectively. Enc and Dec indicated the encoding and decoding processes, respectively. The encoding process involves several layers starting with (1) the input image patch $8 \times 8$; (2) the Conv2D layer that indicates a 2D convolutional layer with 16 filters $3 \times 3$ kernel size, activation "ReLU" and padding "same"; the "ReLU" activation is used due to its simplicity and computation efficiency compared to other activation functions [34]. (3) MAX-Pooling layer that indicates a 2D max-pooling $2 \times 2$ region with padding "same"; (4) Conv2D layer with 8 filters $3 \times 3$ kernel size, activation "ReLU" and padding "same"; (5) Max-Pooling $2 \times 2$ region with padding "same"; and (6) Conv2D layer with 8 filters $3 \times 3$ kernel size, activation "ReLU" and padding "same". The CAEs are fully convolutional networks; thus, the decoding process is including a convolution. The decoding process involves several layers starting with (1) the Conv2D layer that indicates a 2D convolutional layer with 8 filters $3 \times 3$ kernel size, activation "ReLU" and padding 'same'; (2) the UpSampling layer that indicates a 2D UpSampling $2 \times 2$ region; (3) the Conv2D layer with 8 filters $3 \times 3$ kernel size, activation "ReLU" and padding "same"; (4) UpSampling $2 \times 2$ region; (5) the Conv2D layer with 16 filters $3 \times 3$ kernel size, activation "ReLU" and padding "same"; and (6) the Conv2D layer with 1 filter $3 \times 3$ kernel size, activation "linear" and padding "same". Thus, Adadelta optimization is used throughout training, and the MSE between the reconstructed output patches and the target patches $\left\{P_{\mathrm{i}}^{\mathrm{H}}\right\}_{\mathrm{i}=1}^{\mathrm{n}}$ is used for updating the weights as follows:

$$
\mathcal{L}\left(\left\{\tilde{P}_{\mathrm{i}}\right\}_{\mathrm{i}=1}^{\mathrm{n}},\left\{P_{\mathrm{i}}^{\mathrm{H}}\right\}_{\mathrm{i}=1}^{\mathrm{n}}\right)=\frac{1}{2} \sum_{i=1}^{\mathrm{n}}\left\|\tilde{P}_{\mathrm{i}}-P_{\mathrm{i}}^{\mathrm{H}}\right\|_{2}^{2}
$$

After updating the weights, the back-propagation algorithm is utilized for training the convolutional autoencoder network. In the stage of testing, because of similar characteristics between the PAN and the corresponding intensity component of the MS image, the trained network is relied upon to improve the intensity component of MS image; firstly, the intensity component $I$ which is generated by Eq. (5) is partitioned $\left\{I_{\mathrm{i}}\right\}_{\mathrm{i}=1}^{\mathrm{n}}$ and is then fed to the trained network for generating an estimated intensity component $\left\{E_{I_{i}}\right\}_{\mathrm{i}=1}^{\mathrm{n}}$. Thus, the $\left\{E_{I_{i}}\right\}_{\mathrm{i}=1}^{\mathrm{n}}$ is being tiled.

\subsection{Fusion process}

The estimated intensity component $E_{I}$ is employed to enhance the PAN image by using the two-scale guided filter. Firstly, the $E_{I}$ is being used as the guidance image and the PAN image as the input image.

$$
O_{1}=\mathbf{G F}\left(E_{I}, P A N\right)
$$

The difference between the approximation image $O_{1}$ and the input image $E_{I}$ is represented by the spatial detail $D_{1}$. Hence, $D_{1}$ will blend with low-frequency component and may cause serious spectral distortion [35]; therefore, $D_{1}$ is then utilized as the input image for the second scale of guided filter $\mathrm{O}_{2}$.

$$
D_{1}=P A N-O_{1}
$$




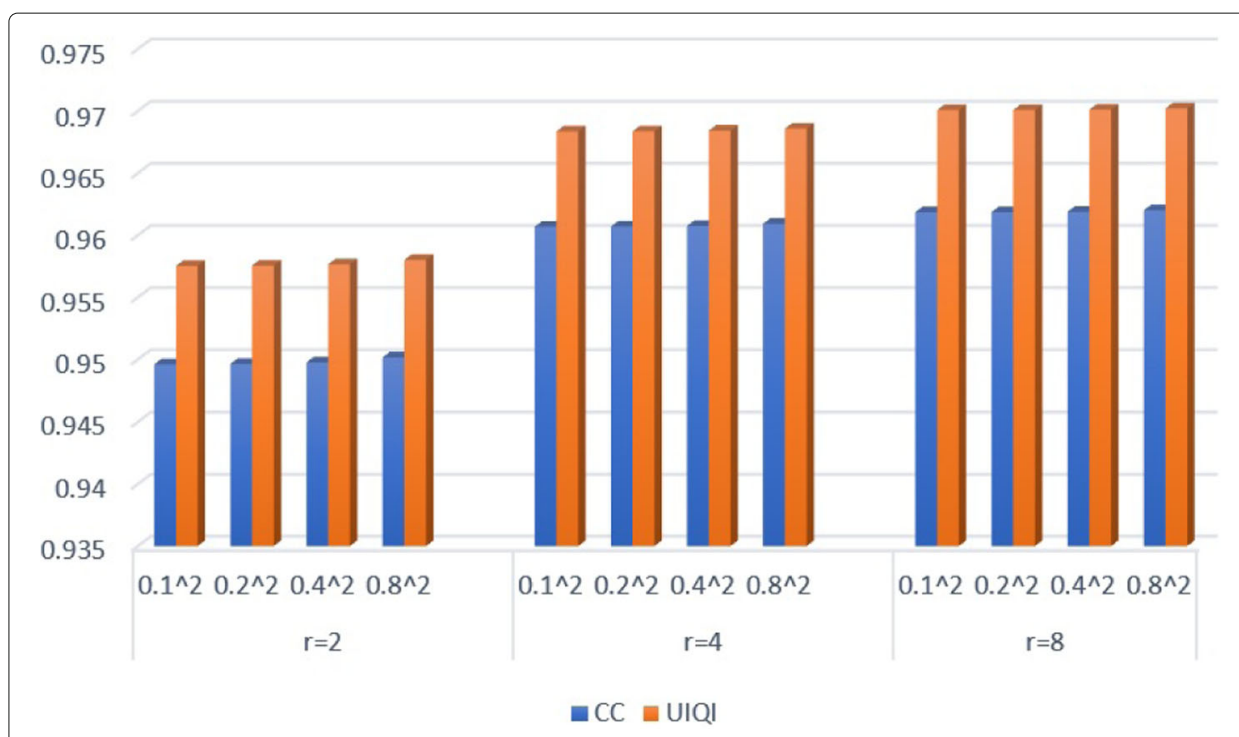

Fig. 3 The influence of the parameters $r$ and $\zeta$ on the fusing result concerning CC and UIQI indexes. Higher $\mathrm{CC}$ and $\mathrm{UIQI}$ indicate the better-fused effect

$$
O_{2}=\mathbf{G F}\left(E_{I}, O_{1}\right)
$$

The difference between $O_{1}$ and $O_{2}$ is represented by the spatial detail $D_{2}$.

$$
D_{2}=O_{1}-O_{2}
$$

The total semantic map $D_{\text {Total }}$ is injected into the upsampled MS image through injection gains $g_{i}$ which are adjusted by (19).

$$
\begin{aligned}
& D_{\text {Total }}=D_{1}+D_{2} \\
& \mathrm{~g}_{\mathrm{i}}=\frac{\operatorname{cov}\left(M S_{i}, E_{I}\right)}{\operatorname{var}\left(E_{I}\right)}
\end{aligned}
$$

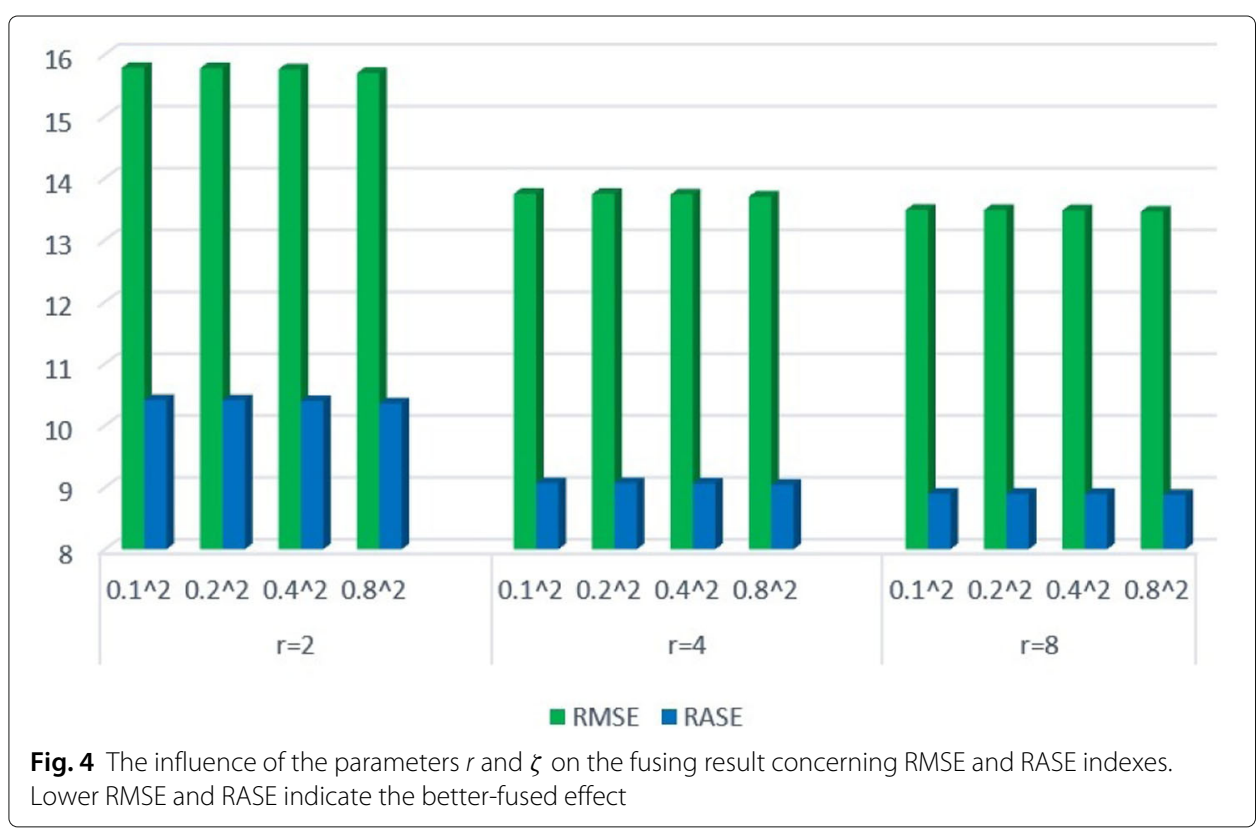




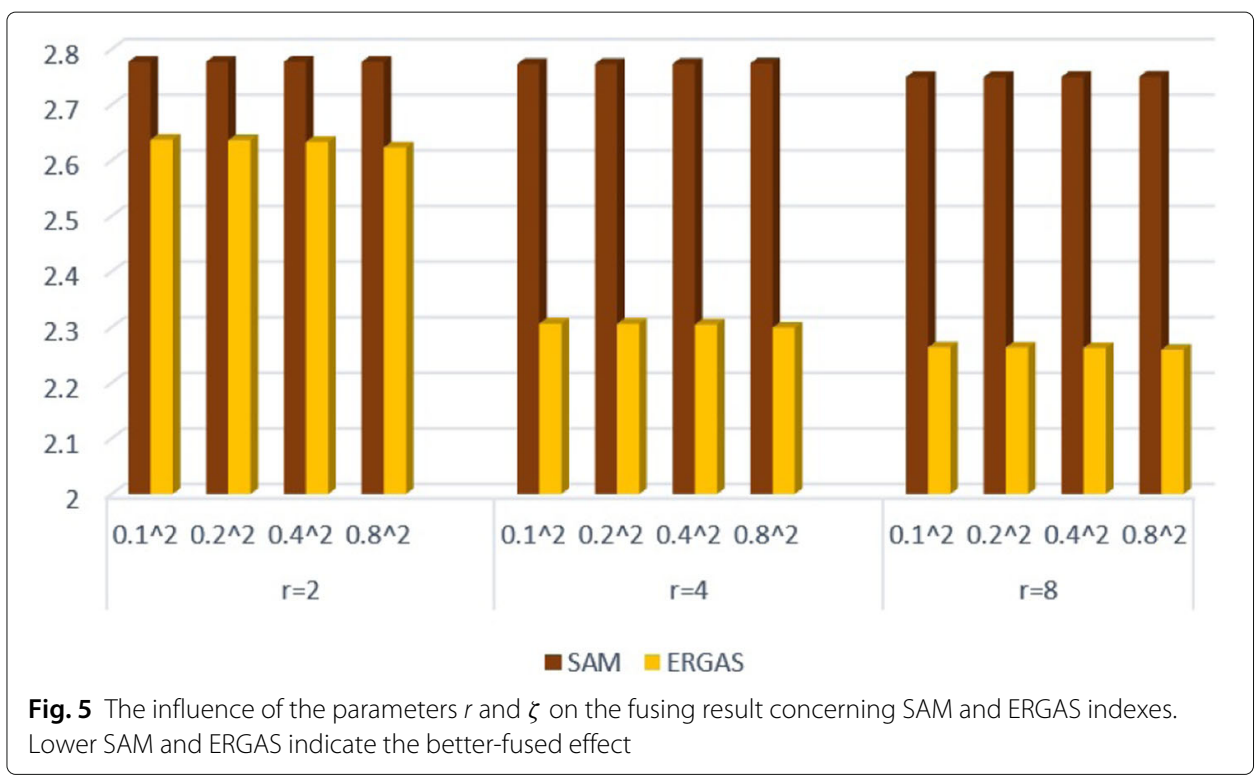

The high-resolution multi-spectral (HRMS) fused image is conducted by the following equation:

HRMS $=M S_{\mathrm{i}}+\mathrm{g}_{\mathrm{i}} D_{\text {Total }}$

\section{Results and discussion}

In this section, several experiments were performed on different datasets to evaluate the performance of the model based on some quality metrics. Here, $8 \times 8$ patches with 5 overlapping pixels of the degraded PAN and the original PAN images that include 500,000 patch pairs were utilized for training the network. In total, six datasets have been selected for implementation purposes. Three degraded datasets (full reference), which means the reference image is available, and three real datasets (no reference image), namely QuickBird and GeoEye.

Therefore, we compared our technique with several conventional efficient pansharpening methods, such as IHS [11], PCA [12], BDSD [36], PRACS [37], and AIHS [31], and several state-of-the-art methods such as SFIM [15], MTF-GLP [16], Indusion [17], MSGF

Table 1 Descriptions of the experimental datasets

\begin{tabular}{|c|c|c|c|}
\hline Datasets & Size & Spatial resolution & Region and date \\
\hline \multirow[t]{2}{*}{ Degraded QuickBird Figs. 6 and 7} & PAN, $256 \times 256$ & $0.7 \mathrm{~m} / 2.8 \mathrm{~m}$ & Sundarbans, India on \\
\hline & MS, $64 \times 64$ & & 21 November 2002 \\
\hline \multirow[t]{2}{*}{ Degraded GeoEye Fig. 8} & PAN, $256 \times 256$ & $0.5 \mathrm{~m} / 2 \mathrm{~m}$ & Hobart, Australia on \\
\hline & $M S, 64 \times 64$ & & 24 February 2009 \\
\hline \multirow[t]{2}{*}{ Real QuickBird Fig. 9} & PAN, $1024 \times 1024$ & $0.7 \mathrm{~m} / 2.8 \mathrm{~m}$ & Xi'an, China on \\
\hline & MS, $256 \times 256$ & & 30 September 2008 \\
\hline \multirow[t]{2}{*}{ Real QuickBird Fig. 10} & PAN, $1024 \times 1024$ & $0.7 \mathrm{~m} / 2.8 \mathrm{~m}$ & Sundarbans, India on \\
\hline & MS, $256 \times 256$ & & 21 November 2002 \\
\hline \multirow[t]{2}{*}{ Real GeoEye Fig. 11} & PAN, $1024 \times 1024$ & $0.5 \mathrm{~m} / 2 \mathrm{~m}$ & Hobart, Australia on \\
\hline & MS, $256 \times 256$ & & 24 February 2009 \\
\hline
\end{tabular}




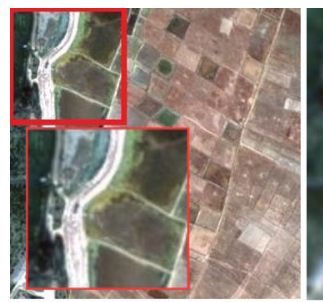

(a)

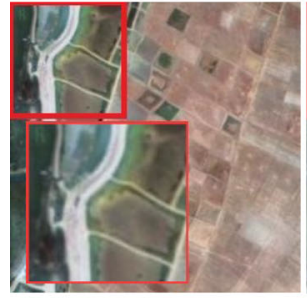

(e)

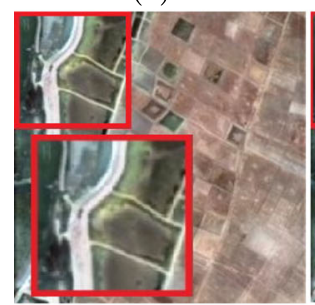

(i)

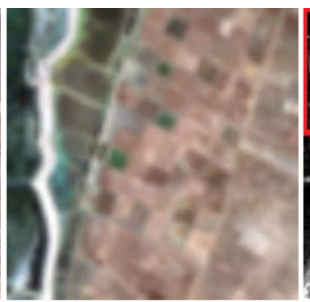

(b)

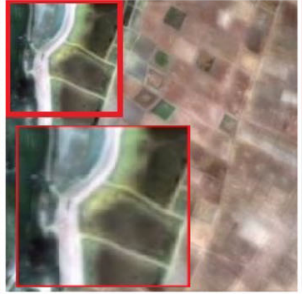

(f)

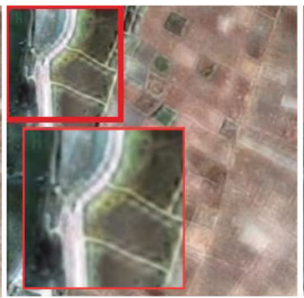

(j)

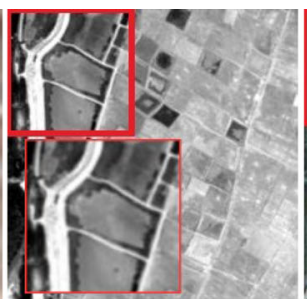

(c)

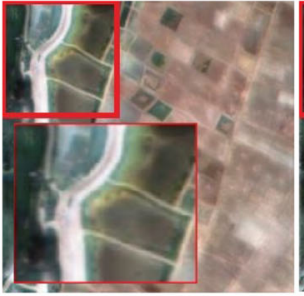

(g)

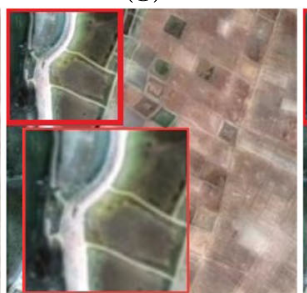

(k)

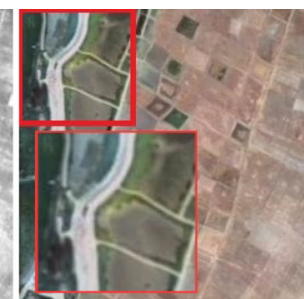

(d)

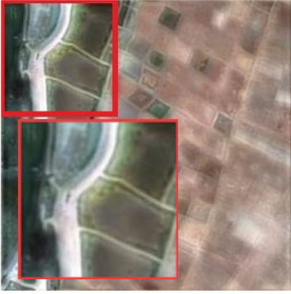

(h)

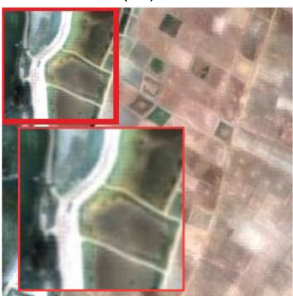

(1)

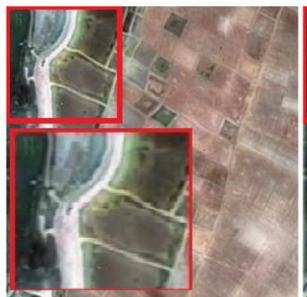

(m)

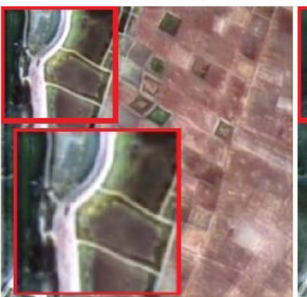

(n)

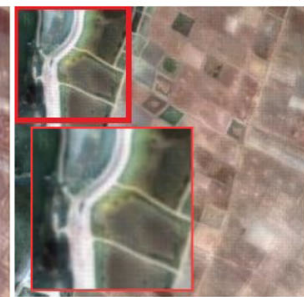

(o)

Fig. 6 Fusion results of the full reference QickBird-1 dataset. a Reference image $(256 \times 256)$. b Degraded MS image $(64 \times 64)$. c PAN image $(256 \times 256)$. d IHS method. e AlHS method. f PCA method. g BDSD method. $\mathbf{h}$ PRACS method. i SFIM method. $\mathbf{j}$ Indusion method. k MTF-GLP method. I CAE method. $\mathbf{m}$ MSGF method. $\mathbf{n}$ PNN method. o Proposed method

[19], CAE [28], and PNN [38]. Moreover, seven image quality indexes are broadly utilized, to assess the quality of the fused image, which are:

1 Correlation coefficient (CC) [39]

2 Universal Image Quality Index (UIQI) [40]

3 Quaternion Theory-based Quality Index (Q4) [40]

4 Root mean square error (RMSE) [41]

5 Relative average spectral error (RASE) [42]

6 Spectral Angle Mapper (SAM) [43]

7 Erreur Relative Globale Adimensionnelle de Synthese (ERGAS) [44]

To assess the quality of the fused images concerning real datasets, $D_{s}, D_{\lambda}$, and QNR [45] were employed. The ideal value of each quality index is shown in parentheses in the tables. 


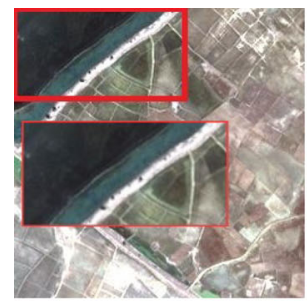

(a)

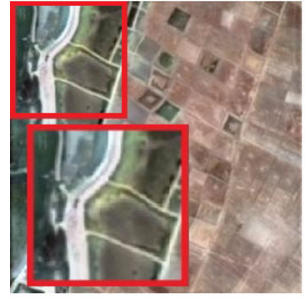

(e)

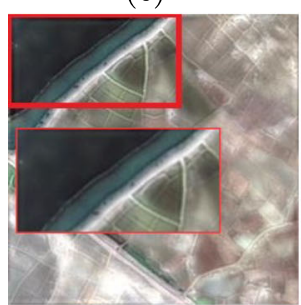

(i)

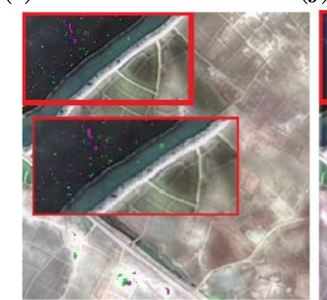

(m)

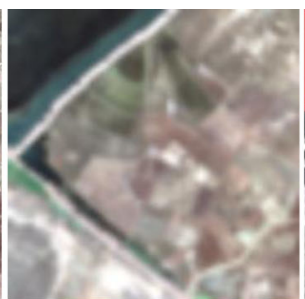

(b)

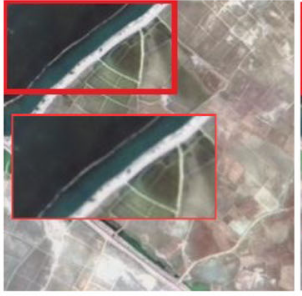

(f)

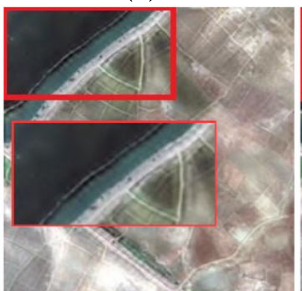

(j)

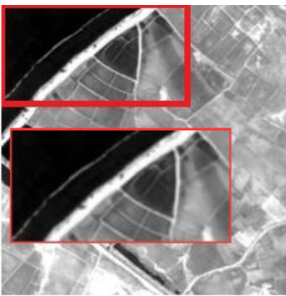

(c)

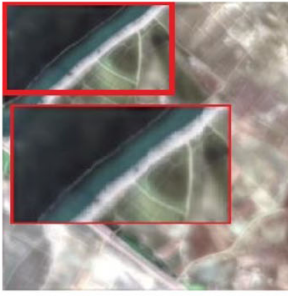

(g)

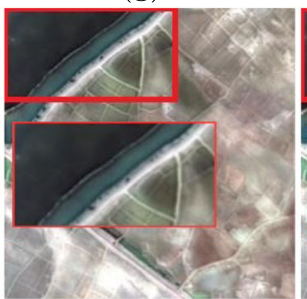

(k)

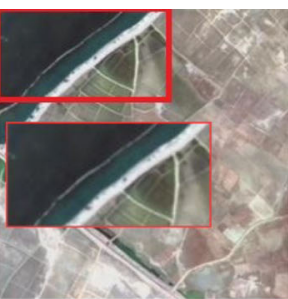

(d)

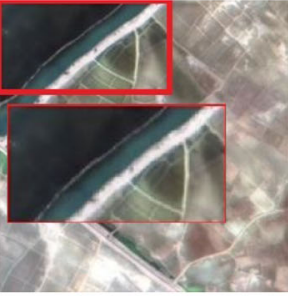

(h)

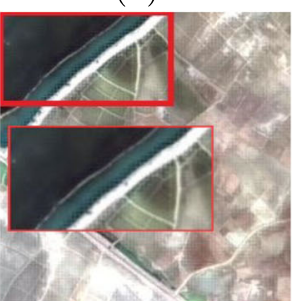

(1)

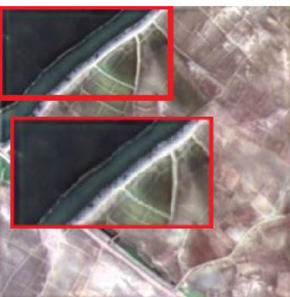

(n)

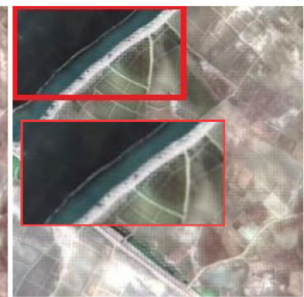

(o)

Fig. 7 Fusion results of the full reference QickBird- 2 dataset. a Reference image $(256 \times 256)$. b Degraded MS image $(64 \times 64)$. c PAN image $(256 \times 256)$. d IHS method. e AlHS method. f PCA method. g BDSD method. $\mathbf{h}$ PRACS method. i SFIM method. $\mathbf{j}$ Indusion method. k MTF-GLP method. I CAE method. $\mathbf{m}$ MSGF method. $\mathbf{n}$ PNN method. o Proposed method

\subsection{Parameter investigation}

Here, we study the influence of parameter setting in the guided filter on the fusion simulation of degraded QuickBird-1 dataset, namely, window size $r$ and the regularization parameter $\zeta$. Figures 3, 4, and 5 illustrate the influence of these parameters, where the horizontal axis is the regularization parameter $\zeta$ concerning three cases of window size $r$ and the vertical axis is quality index results. Therefore, as can be seen, the best performance results originated from setting the parameters $r$ and $\zeta$ at 8 and $0.8^{2}$, respectively.

\subsection{Fusion results of degraded datasets (full reference)}

In this section, the simulations were carried out on degraded datasets that have the reference image to evaluate our proposed method according to Wald's protocol [46]. Regarding the degraded datasets (QuickBird, GeoEye), the sizes of the MS image and the PAN image 
Table 2 Numerical results of the full reference QuickBird-1 dataset

\begin{tabular}{llllllll}
\hline Method & CC(1) & UIQI(1) & RMES(0) & RASE(0) & SAM(0) & ERGAS(0) & Q4(1) \\
\hline IHS & 0.91446 & 0.93471 & 18.509 & 12.673 & 3.837 & 3.2713 & 0.8211 \\
AlHS & 0.93518 & 0.95778 & 15.045 & 10.301 & 3.7497 & 2.7063 & 0.88383 \\
PCA & 0.93743 & 0.94871 & 16.408 & 11.234 & 3.6063 & 2.9025 & 0.84805 \\
BDSD & 0.92229 & 0.95204 & 16.435 & 11.253 & 3.7728 & 2.9444 & 0.86477 \\
PRACS & 0.93038 & 0.95991 & 14.992 & 10.265 & 3.8317 & 2.7466 & 0.86389 \\
SFIM & 0.89958 & 0.92403 & 20.297 & 13.897 & 3.6792 & 3.5142 & 0.81325 \\
Indusion & 0.91806 & 0.93911 & 17.967 & 12.302 & 3.5524 & 3.1483 & 0.81981 \\
MTF-GLP & 0.91144 & 0.93984 & 18.306 & 12.534 & 3.6399 & 3.2217 & 0.84308 \\
CAE & 0.94467 & 0.95735 & 23.955 & 16.402 & 3.3501 & 4.1479 & $\mathbf{0 . 8 9 6 8}$ \\
MSGF & 0.9264 & 0.93907 & 18.165 & 12.437 & 3.3836 & 3.1604 & 0.88853 \\
PNN & 0.89955 & 0.92229 & 21.857 & 14.965 & 4.8681 & 3.7866 & 0.8183 \\
Proposed & $\mathbf{0 . 9 4 5 6 3}$ & $\mathbf{0 . 9 6 3 5 5}$ & $\mathbf{1 4 . 0 9 6}$ & $\mathbf{9 . 6 5 1 4}$ & $\mathbf{3 . 3 2 6 4}$ & $\mathbf{2 . 5 3 5 7}$ & 0.88685 \\
\hline
\end{tabular}

are $64 \times 64$ and $256 \times 256$, respectively. The descriptions of the experimental datasets are shown in Table 1.

\subsubsection{Experiments on degraded QuickBird datasets}

In this section, two pairs of QuickBird satellite datasets were examined; Fig. 6 illustrates the fusion results of the degraded QuickBird-1 dataset. For better comparison, the red square area is enlarged and then displayed at the bottom left of the fusion image. As can be observed, Fig. $6 \mathrm{~d}-\mathrm{j}$ methods have more inferior pansharpening results than CAE and proposed methods.

Figure $6 \mathrm{i}-\mathrm{j}$ suffer from spatial distortion. Figure $6 \mathrm{~m}$ suffers from spatial and spectral distortions. The fusion result of the PNN method is depicted in Fig. 6n, which produces some unnatural color compared with the reference image. Furthermore, Fig. $6 \mathrm{l} \mathrm{CAE}$ and proposed method Fig. 6o look most similar to the reference image Fig. 6a, but the proposed method performs better in terms of spectral and spatial fidelity. Similar observations can be made regarding the experimental results from the QuickBird-2 dataset. Figure 7 displays the fusion results of the degraded QuickBird-2 dataset. For better visual comparison, the red rectangle area is enlarged and then displayed at the bottom of the selected area; thus, the proposed and CAE methods have performed better visual effects.

Table 3 Numerical results of the full reference QuickBird-2 dataset

\begin{tabular}{llllllll}
\hline Method & CC(1) & UIQI(1) & RMES(0) & RASE(0) & SAM(0) & ERGAS(0) & Q4(1) \\
\hline IHS & 0.94819 & 0.95489 & 16.013 & 10.565 & 2.9752 & 2.6898 & 0.85877 \\
AIHS & 0.96171 & 0.96337 & 13.465 & 9.8843 & 2.8699 & 2.2927 & 0.8984 \\
PCA & 0.95507 & 0.95906 & 15.256 & 10.066 & 2.9418 & 2.557 & 0.84805 \\
BDSD & 0.9425 & 0.9547 & 16.41 & 10.828 & 3.0727 & 2.7548 & 0.85536 \\
PRACS & 0.96067 & 0.97082 & 13.29 & 8.7685 & 3.0473 & 2.2505 & 0.89358 \\
SFIM & 0.92309 & 0.9339 & 19.612 & 12.94 & 3.0571 & 3.2638 & 0.82182 \\
Indusion & 0.93623 & 0.94457 & 17.754 & 11.714 & 2.9083 & 2.9657 & 0.82554 \\
MTF-GLP & 0.93661 & 0.94967 & 17.309 & 11.42 & 2.9938 & 2.9018 & 0.85643 \\
CAE & 0.96082 & 0.96583 & 18.999 & 12.535 & 2.871 & 3.1639 & 0.8957 \\
MSGF & 0.9372 & 0.94047 & 18.635 & 12.295 & 2.9182 & 3.1012 & 0.90274 \\
PNN & 0.92756 & 0.93768 & 19.765 & 13.041 & 3.8949 & 3.2934 & 0.84088 \\
Proposed & $\mathbf{0 . 9 6 3 6 6}$ & $\mathbf{0 . 9 7 1 5 7}$ & $\mathbf{1 3 . 2 8 6}$ & $\mathbf{8 . 7 6 5 7}$ & $\mathbf{2 . 7 6 8 6}$ & $\mathbf{2 . 2 2 7 1}$ & $\mathbf{0 . 9 0 6 1 7}$ \\
\hline
\end{tabular}




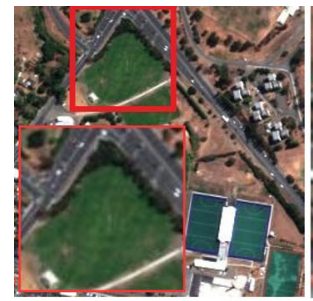

(a)

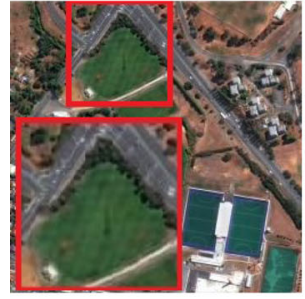

(e)

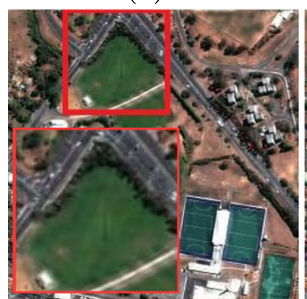

(i)

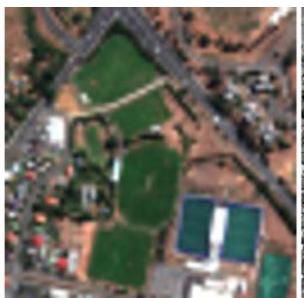

(b)

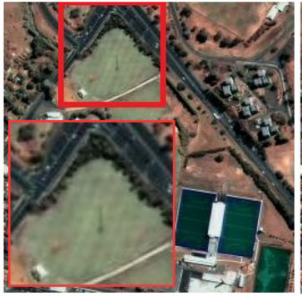

(f)

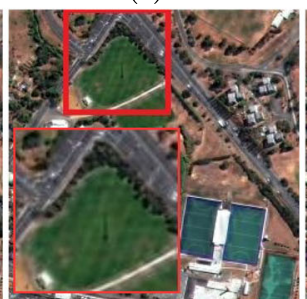

(j)

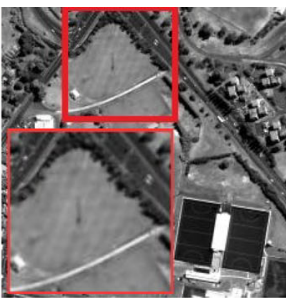

(c)

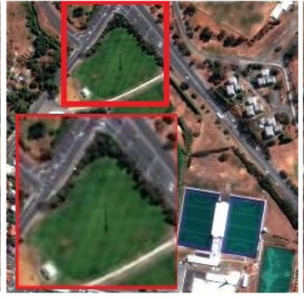

(g)

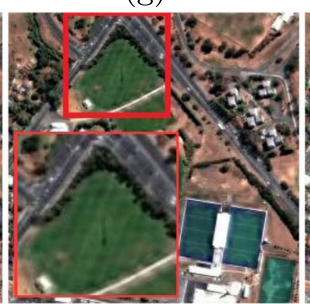

(k)

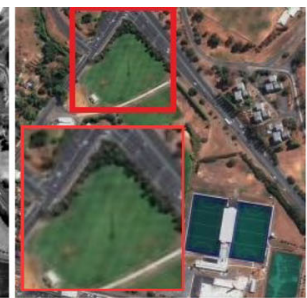

(d)

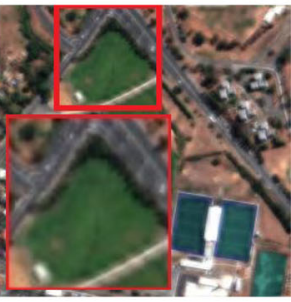

(h)

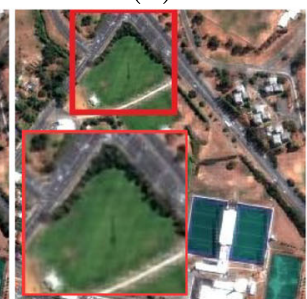

(l)

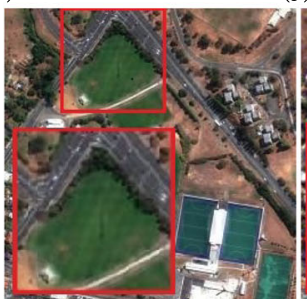

(m)

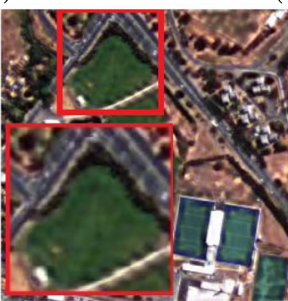

(n)

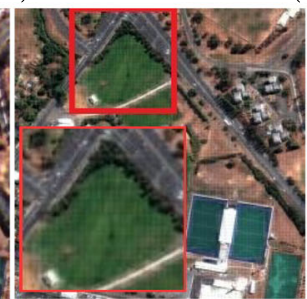

(o)

Fig. 8 Fusion results of the full reference GeoEye-1 dataset. a Reference image $(256 \times 256)$. b Degraded MS image $(64 \times 64)$. c PAN image $(256 \times 256)$. d IHS method. e AlHS method. f PCA method. g BDSD method. $\mathbf{h}$ PRACS method. i SFIM method. $\mathbf{j}$ Indusion method. k MTF-GLP method. I CAE method. $\mathbf{m}$ MSGF method. $\mathbf{n}$ PNN method. o Proposed method

Table 4 Numerical results of the full reference GeoEye-1 dataset

\begin{tabular}{llllllll}
\hline Method & $\mathbf{C C}(\mathbf{1})$ & UIQI(1) & RMES(0) & RASE(0) & SAM(0) & ERGAS(0) & Q4(1) \\
\hline IHS & 0.94344 & 0.94327 & 19.622 & 18.015 & 5.6283 & 4.6538 & 0.8837 \\
AlHS & 0.95117 & 0.95566 & 17.432 & 16.004 & 5.2561 & 4.0669 & 0.90041 \\
PCA & 0.71449 & 0.80704 & 36.19 & 33.227 & 14.476 & 8.9614 & 0.68996 \\
BDSD & 0.95674 & 0.96131 & 17.53 & 16.095 & 6.2016 & 4.0897 & 0.91719 \\
PRACS & 0.94091 & 0.9545 & 17.857 & 16.395 & 4.6631 & 4.2619 & 0.89865 \\
SFIM & 0.7315 & 0.6906 & 57.368 & 52.671 & 6.0017 & 14.559 & 0.89337 \\
Indusion & 0.94438 & 0.94489 & 20.182 & 18.529 & 5.8298 & 4.4905 & 0.88997 \\
MTF-GLP & 0.95403 & 0.95517 & 18.87 & 17.325 & 6.6894 & 4.248 & 0.90944 \\
CAE & 0.95082 & 0.94401 & 26.323 & 24.168 & 5.389 & 5.9969 & 0.8993 \\
MSGF & 0.9575 & 0.9571 & 16.217 & 14.791 & $\mathbf{4 . 6 0 1 7}$ & 3.7496 & 0.92439 \\
PNN & 0.93825 & 0.91003 & 27.623 & 25.361 & 9.9312 & 5.6757 & 0.86618 \\
Proposed & $\mathbf{0 . 9 5 7 9 4}$ & $\mathbf{0 . 9 6 3 7 6}$ & $\mathbf{1 6 . 1 0 3}$ & $\mathbf{1 4 . 7 8 4}$ & 4.8976 & $\mathbf{3 . 7 1 4 5}$ & $\mathbf{0 . 9 1 7 2 8}$ \\
\hline
\end{tabular}




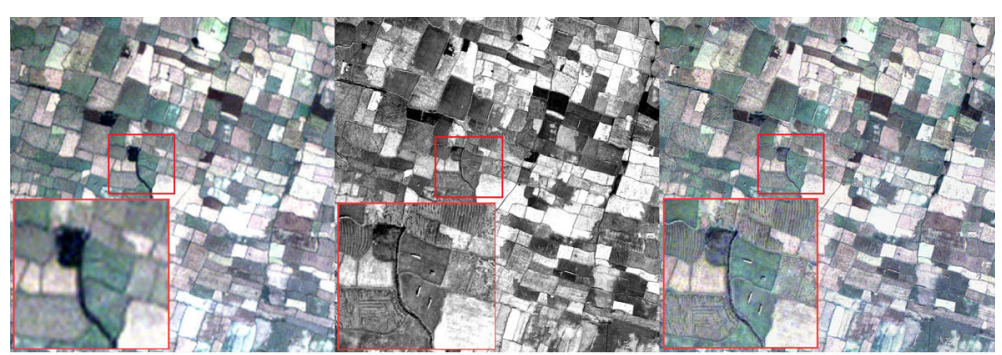

(a)

(b)

(c)

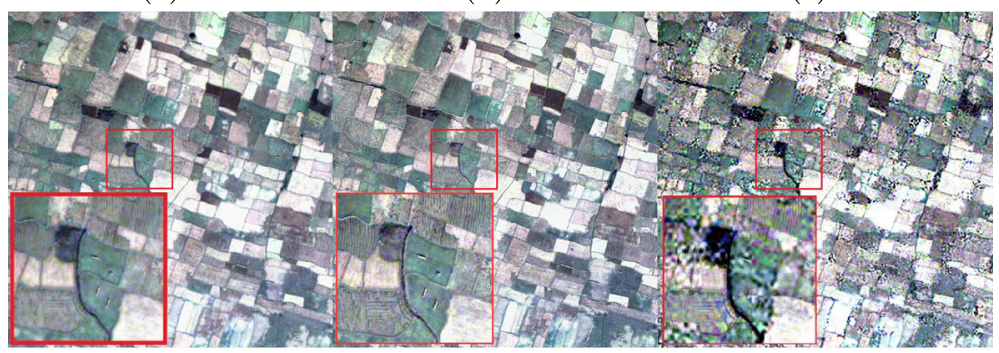

(d)

(e)

(f)

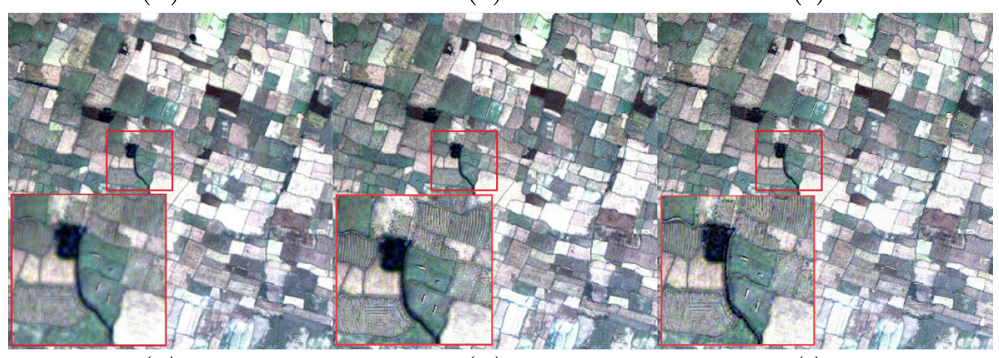

(g)

(h)

(i)

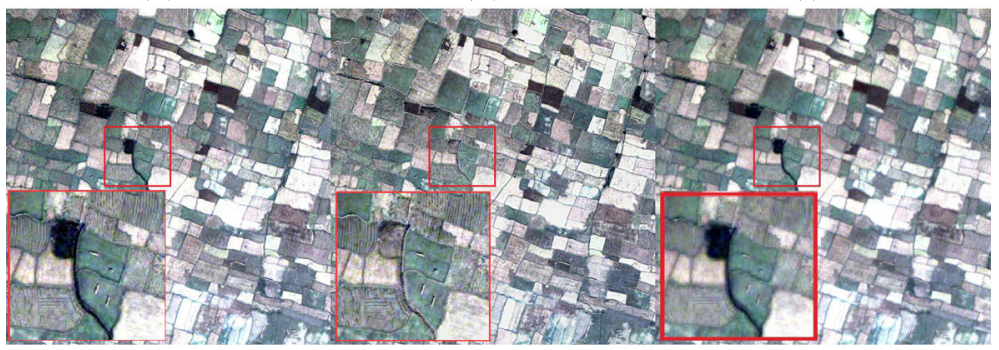

(j)

(k)

(1)

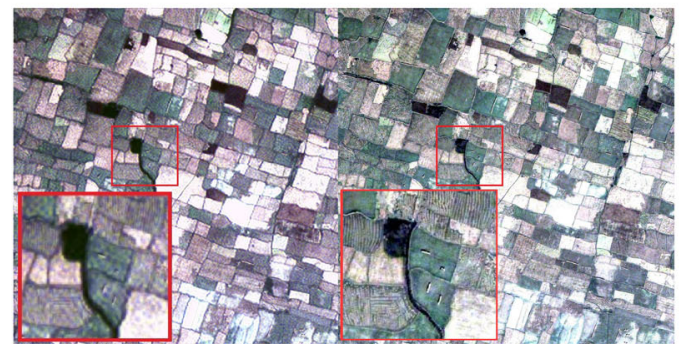

$(\mathrm{m})$

(n)

Fig. 9 Fusion results of the real QuickBird-1 dataset. a Upsampled MS image $(1024 \times 1024)$. b PAN image $(1024 \times 1024)$. c $1 H S$ method. d AlHS method. e PCA method. f BDSD method. g PRACS method. h SFIM method. i Indusion method. j MTF-GLP method. $\mathbf{k}$ CAE method. I MSGF method. $\mathbf{m}$ PNN method. $\mathbf{n}$ Proposed method

In terms of objective evaluation, the numerical indexes of fused images for Figs. 6 and 7 are computed and reported in Tables 2 and 3, respectively. From both tables, it is clear that our method can contribute to the best values in terms of quality indexes. 


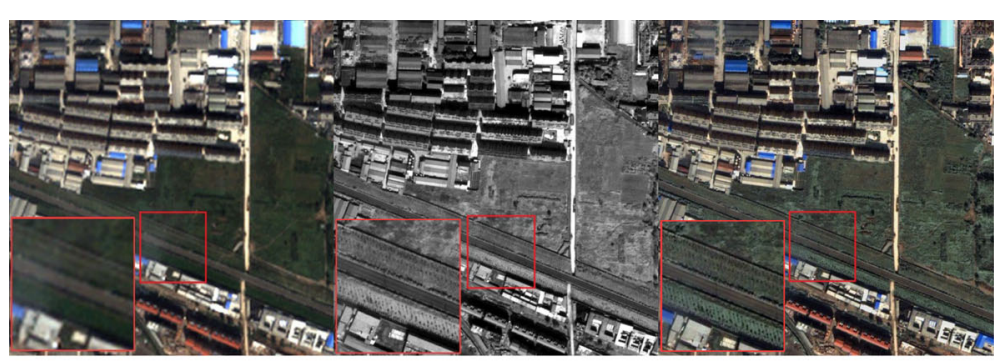

(a)

(c)

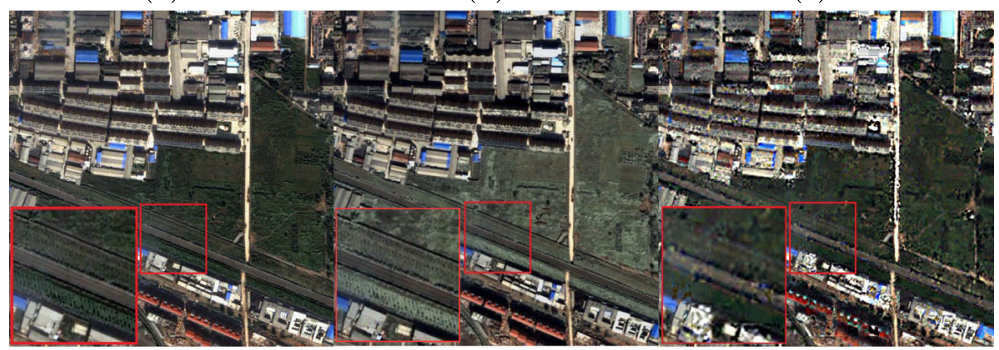

(d)

(e)

(f)

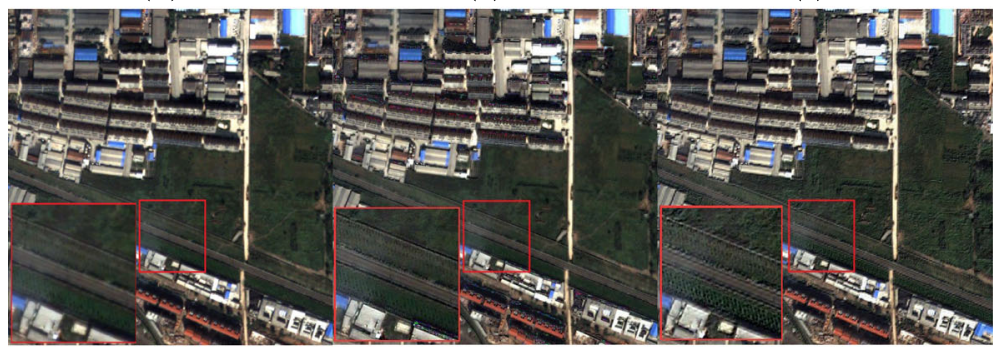

(g)

(h)

(i)

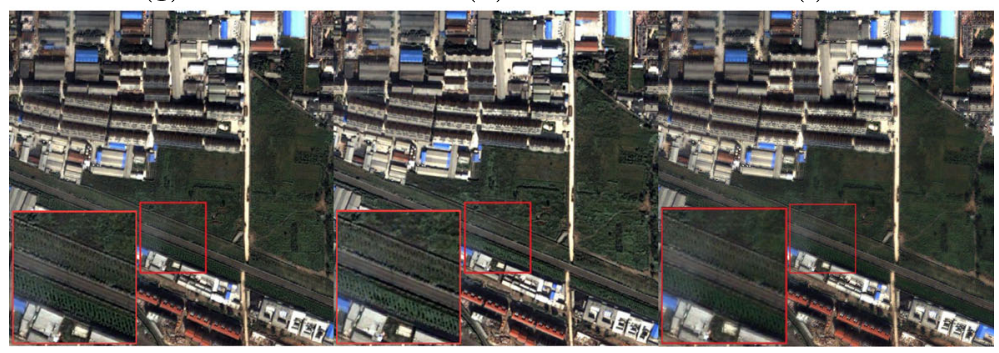

(j)

(k)

(l)

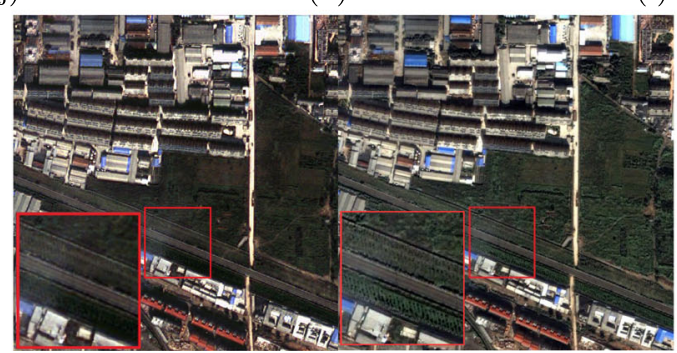

(m)

(n)

Fig. 10 Fusion results of the real QuickBird-2 dataset. a Upsampled MS image (1024× 1024). b PAN image (1024× 1024). c $1 H S$ method. d AIHS method. e PCA method. f BDSD method. g PRACS method. h SFIM method. i Indusion method. j MTF-GLP method. k CAE method. I MSGF method. $\mathbf{m}$ PNN method. $\mathbf{n}$ Proposed method 
Table 5 Numerical results of the real QuickBird-1 dataset

\begin{tabular}{llll}
\hline Method & $\boldsymbol{D}_{\boldsymbol{s}}(\mathbf{0})$ & $\boldsymbol{D}_{\boldsymbol{\lambda}}(\mathbf{0})$ & $\mathbf{Q N R}(\mathbf{1})$ \\
\hline Proposed & $\mathbf{0 . 1 2 8 9}$ & $\mathbf{0 . 0 0 0 8}$ & $\mathbf{0 . 9 6 9 9}$ \\
PNN & 0.1957 & 0.2305 & 0.8976 \\
MSGF & 0.151 & 0.019 & 0.9603 \\
CAE & 0.16027 & 0.001 & 0.9620 \\
MTF-GLP & 0.15097 & 0.068214 & 0.94468 \\
Indusion & 0.13001 & 0.042951 & 0.95876 \\
SFIM & 0.13109 & 0.052531 & 0.95375 \\
PRACS & 0.1834 & 0.0382 & 0.9469 \\
BDSD & 0.1998 & 0.1235 & 0.92189 \\
IHS & 0.24386 & 0.06756 & 0.91959 \\
AlHS & 0.22026 & 0.043312 & 0.93374 \\
PCA & 0.24261 & 0.05516 & 0.92611 \\
\hline
\end{tabular}

\subsubsection{Experiment on degraded GeoEye dataset}

Figure 8 displays the fusion results of the degraded GeoEye- 1 dataset. The red square area is enlarged and then displayed at the bottom left of the fusion image. As shown in Fig. 8f, PCA produced seedy color in the fused image, and Fig. $8 \mathrm{f}-\mathrm{h}$ suffer from the spectral distortion. Here, it can be seen that the SFIM, Indusion, and MTF-GLP methods perform well, as shown in Fig. 8i-k. We can also observe from Fig. 81 that the result of the CAE method has a color problem at the vegetation area compared with the reference image. The colors of the fusion image for MSGF and PNN methods have remarkable distortion, as shown in Fig. 8m, n. Overall, the proposed method created the fused image, with appropriate spectral and spatial resolution, as shown in Fig. 80 compared with others.

The numerical indexes of fused images for Fig. 8 are computed and reported in Table 4 . From the table, it is clear that our method can contribute to the best values in the most quality indexes.

\subsection{Fusion results of real datasets (no reference)}

Regarding real datasets, two kinds of real datasets (QuickBird, GeoEye) were implemented, and the sizes of the MS image and the PAN image are $256 \times 256$ and $1024 \times 1024$, respectively.

Table 6 Numerical results of the real QuickBird-2 dataset

\begin{tabular}{llll}
\hline Method & $\boldsymbol{D}_{\boldsymbol{s}}(\mathbf{0})$ & $\boldsymbol{D}_{\boldsymbol{\lambda}}(\mathbf{0})$ & $\mathbf{Q N R}(\mathbf{1})$ \\
\hline Proposed & 0.13935 & $\mathbf{0 . 0 8 1 3 1 9}$ & $\mathbf{0 . 9 6 5 9 8}$ \\
PNN & 0.181486 & 0.095215 & 0.96407 \\
MSGF & 0.082709 & 0.102 & 0.96379 \\
CAE & 0.19834 & 0.093565 & 0.94344 \\
MTF-GLP & 0.13929 & 0.092786 & 0.93708 \\
Indusion & $\mathbf{0 . 0 3 0 6 6 9}$ & 0.087048 & 0.96348 \\
SFIM & 0.066346 & 0.14364 & 0.93699 \\
PRACS & 0.124 & 0.09274 & 0.9646 \\
BDSD & 0.3088 & 0.0974 & 0.9593 \\
IHS & 0.2534 & 0.10823 & 0.91008 \\
AlHS & 0.16242 & 0.095591 & 0.93691 \\
PCA & 0.28645 & 0.13137 & 0.90127 \\
\hline
\end{tabular}




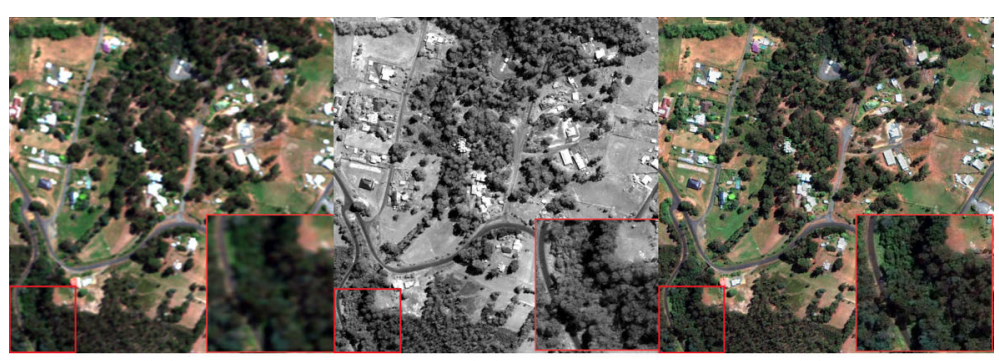

(a)

(b)

(c)

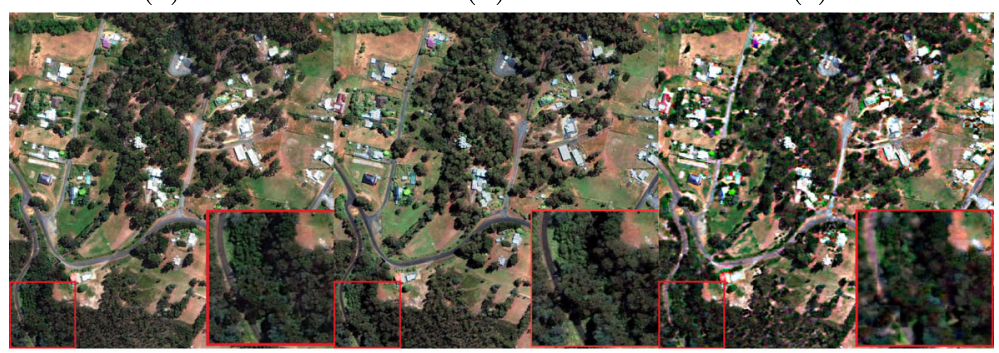

(d)

(e)

(f)

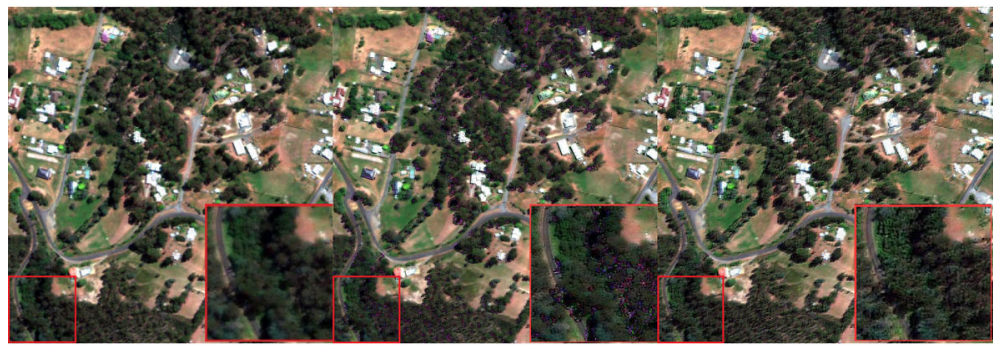

(g)

(h)

(i)

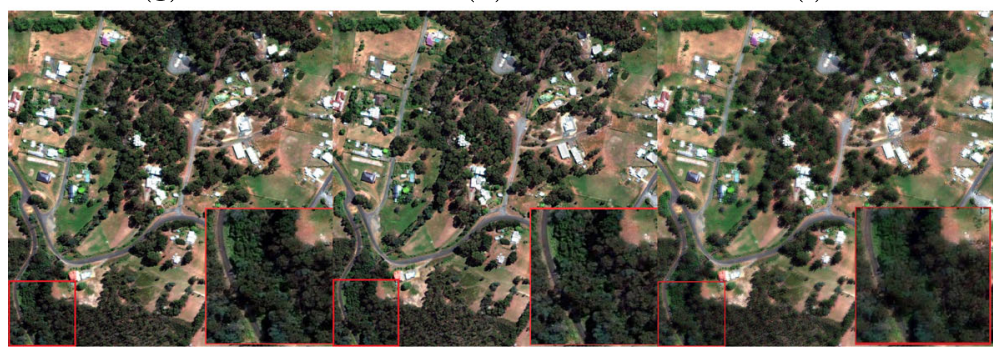

(j)

(k)

(l)

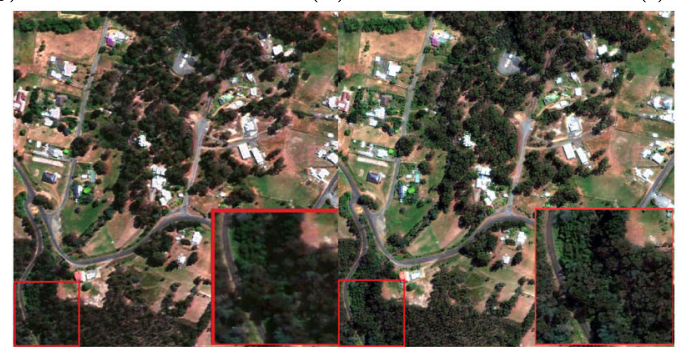

$(\mathrm{m})$

(n)

Fig. 11 Fusion results of the real GeoEye-1 dataset. a Upsampled MS image (1024×1024). b PAN image (1024× 1024). c $1 H S$ method. d AlHS method. e PCA method. f BDSD method. g PRACS method. h SFIM method. i Indusion method. j MTF-GLP method. $\mathbf{k}$ CAE method. I MSGF method. $\mathbf{m}$ PNN method. $\mathbf{n}$ Proposed method 


\subsubsection{Experiments on real QuickBird datasets}

Two pairs of real QuickBird satellite datasets were examined; for better visual comparison, the red square area is enlarged and then displayed at the bottom left of the fusion image. Figure 9 displays the fusion results of real QuickBird-1 dataset.

The fusion results of all methods improved, but the CS-based method and CAE method suffer from spectral distortion, as shown in Fig. 9c, e, and k. The BDSD fusion method has remarkable distortions. For SFIM, Indusion, and MTF-GLP methods, they can achieve relatively better results regarding spectral resolution than others, as shown in Fig. $9 \mathrm{~h}-\mathrm{j}$. The MSGF method suffers from spatial distortion, as shown in Fig. 9l, and the colors of the fusion image for the PNN method have remarkable distortions. However, the fusion result of the proposed method can perform better than others, as shown in Fig. 9o. Similarly, the observations can be done regarding the experimental results from the real QuickBird-2 dataset. Figure 10 displays the fusion results of the real QuickBird-2 dataset. The CS-based methods suffer from spectral distortion, as shown in Fig. 10c, e. The BDSD fusion method has remarkable distortions as shown in Fig. 10e. The CAE method can achieve well concerning the spatial aspect but still has a lighter color in the vegetation area compared with the upsampled MS image, as shown in Fig. 10k.

The fusion results of SFIM, Indusion, MTF-GLP, MSGF, PNN, and proposed methods improved in both aspects of spectral and spatial.

The numerical measurements of real data fused images for Figs. 9 and 10 are computed and listed in Tables 5 and 6, respectively.

Table 5 illustrates the proposed method performed the best value in terms of $D_{\lambda}$ and $D_{s}$. Thus, our method showed the best value in terms of $D_{\lambda}$ and QNR, as reported in Table 6 .

\subsubsection{Experiment on real GeoEye dataset}

Figure 11 displays the fusion results of the real GeoEye-1 dataset. The selected red square area is enlarged and then displayed at the bottom right of the fusion image for better visual comparison. As shown in Fig. 11c-e, these methods can perform well regarding spatial aspect but suffer from spectral distortion, and Fig. 11f-i and l, suffer from notable spectral and spatial distortion. Here, it can be seen that the MTF-GLP, CAE, and proposed methods perform well, as shown in Fig. 11j, k, and o.

Overall, the proposed method created the fused image, with appropriate spectral and spatial resolution.

Table 7 Numerical results of the real GeoEye-1 dataset

\begin{tabular}{llll}
\hline Method & $\boldsymbol{D}_{\boldsymbol{s}}(\mathbf{0})$ & $\boldsymbol{D}_{\boldsymbol{\lambda}}(\mathbf{0})$ & $\mathbf{Q N R ( \mathbf { 1 } )}$ \\
\hline Proposed & 0.1083 & 0.0806 & $\mathbf{0 . 9 5 7 0 6}$ \\
PNN & 0.094048 & $\mathbf{0 . 0 6 0 5 5 3}$ & 0.95616 \\
MSGF & 0.1102 & 0.095 & 0.95434 \\
CAE & 0.13473 & 0.14395 & 0.93975 \\
MTF-GLP & 0.12752 & 0.13915 & 0.92936 \\
Indusion & 0.050782 & 0.11372 & 0.9568 \\
SFIM & 0.13797 & 0.20061 & 0.91727 \\
PRACS & $\mathbf{0 . 0 4 6 4}$ & 0.0936 & 0.9566 \\
BDSD & 0.1242 & 0.1269 & 0.9446 \\
IHS & 0.1593 & 0.1277 & 0.92838 \\
AlHS & 0.123 & 0.123 & 0.9452 \\
PCA & 0.1869 & 0.1555 & 0.92199 \\
\hline
\end{tabular}


The numerical indexes of fused images for Fig. 11 are computed and reported in Table 7. From Table 7, the PNN method can perform the best value in terms of $D_{\lambda}$, followed by our method. Overall, our method can still contribute to the best values concerning quality indexes.

\title{
4 Conclusion
}

In this paper, we have proposed a pansharpening technique based on a convolutional autoencoder with AIHS and a multi-scale guided filter. The proposed method first trained the convolutional autoencoder to learn the relationship between the panchromatic image and its degraded version. The trained network is used to enhance the intensity component. Furthermore, the multi-scale guided filter is used to enhance the original panchromatic image. Several experiments were conducted, and the article has put in place the results of the experiment. The outcomes of this research are, first, in terms of visual aspect, the proposed method includes more of the spectral detail of the MS image and spatial detail of the panchromatic image than existing fusion methods. Second, the quality indexes of our method show significant enhancements compared with comparative methods. Overall, the model developed in this research was able to preserve appropriate spatial and spectral aspects of fusion image compared with comparative methods in both aspects, subjective and objective evaluations.

\begin{abstract}
Abbreviations
PAN: Panchromatic image; MS: Multi-spectral image; CNN: Convolutional neural network; AlHS: Adaptive intensity-hue-saturation; CS: Component substitution; MRA: Multi-resolution analysis; PCA: Principal component analysis; GS: Gram-Schimidt; BT: Brovey transform; SFIM: Smoothing Filter-based Intensity Modulation; MTF-GLP: Generalized Laplacian pyramid; MSGF: Multi-scale guided filer; RCNN: Residual convolutional neural network; CAE: Convolutional autoencoder; GF: Guided filter; BDSD: Band-dependent spatial-detail; PRACS: Partial replacement adaptive CS; PNN: Pansharpening by convolutional neural networks. CC: Correlation coefficient; UIQI: Universal Image Quality Index; RMSE: Root mean square error; RASE: Relative average spectral error; SAM: Spectral Angle Mapper; ERGAS: Erreur Relative Globale Adimensionnelle de Synthese. $D_{s}$ : Spatial distortion; $D_{\lambda}$ : Spectral distortion; QNR: Quality with no reference
\end{abstract}

\section{Acknowledgements}

No other acknowledgments.

\section{Authors' contributions}

AAL and YS conceptualized and carried out the implementation; AAL, KZ, and AM wrote and reviewed the paper; AS and MW were in charge of the overall research and contributed to the paper writing; YS contributed to funding acquisition. All authors have read and agreed to the published version of the manuscript.

\section{Funding}

This work was supported by the National Natural Science Foundation of China (Nos. 61771380, 61906145, U1730109, $91438103,61771376,61703328,91438201$, U1701267, 61703328), the Equipment pre-research project of the 13th Five-Years Plan (Nos. 6140137050206, 414120101026, 6140312010103, 6141A020223, 6141B06160301, 6141B07090102), the Major Research Plan in Shaanxi Province of China (Nos. 2017ZDXM-GY-103,017ZDCXL-GY-03-02), the Foundation of the State Key Laboratory of CEMEE (Nos. 2017K0202B, 2018K0101B 2019K0203B, 2019Z0101B), and the Science Basis Research Program in Shaanxi Province of China (Nos. 16JK1823, 2017JM6086, 2019JQ-663).

Availability of data and materials

The datasets used and analyzed during the current study are available from the corresponding author on reasonable request.

\section{Declarations}

Competing interests

The authors declare that they have no competing interests.

\section{Author details}

${ }^{1}$ School of Artificial Intelligence, Xidian University, No. 2 South Taibai Road, 710071 Xian, China. ${ }^{2}$ School of Information Science and Engineering, Shandong Normal University, Jinan 250358 Shandong, China. ${ }^{3}$ Key Laboratory of Radar Signal Processing, Xidian University, No. 2 South Taibai Road, Xian, China. 
Received: 20 October 2020 Accepted: 10 June 2021

Published online: 19 July 2021

\section{References}

1. K. Zhang, M. Wang, S. Yang, L. Jiao, Spatial-spectral-graph-regularized low-rank tensor decomposition for multispectral and hyperspectral image fusion. IEEE J. Sel. Top. Appl. Earth Obs. Remote Sens. 11(4), 1030-1040 (2018)

2. A. Al Smadi, A. Abugabah, in Proceedings of the 2018 the 2 nd International Conference on Video and Image Processing, Intelligent information systems and image processing: a novel pan-sharpening technique based on multiscale decomposition, (2018), pp. 208-212

3. F. Zhang, K. Zhang, Superpixel guided structure sparsity for multispectral and hyperspectral image fusion over couple dictionary. Multimedia Tools Appl. 79(7), 4949-4964 (2020)

4. J. Xu, H. Zhao, P. Yin, D. Jia, G. Li, Remote sensing classification method of vegetation dynamics based on time series Landsat image: a case of opencast mining area in China. EURASIP J. Image Video Process. 2018(1), 113 (2018)

5. A. Alsmadi, S. Yang, K. Zhang, Pansharpening via deep guided filtering network. Int. J. Image Process. Vis. Commun. 5, 1-8 (2018)

6. G. Vivone, L. Alparone, J. Chanussot, M. Dalla Mura, A. Garzelli, G. A. Licciardi, R. Restaino, L. Wald, A critical comparison among pansharpening algorithms. IEEE Trans. Geosci. Remote Sens. 53(5), 2565-2586 (2014)

7. L. Alparone, L. Wald, J. Chanussot, C. Thomas, P. Gamba, L. M. Bruce, Comparison of pansharpening algorithms: outcome of the 2006 GRS-S data-fusion contest. IEEE Trans. Geosci. Remote Sens. 45(10), 3012-3021 (2007)

8. A. Mookambiga, V. Gomathi, Comprehensive review on fusion techniques for spatial information enhancement in hyperspectral imagery. Multidim. Syst. Sign. Process. 27(4), 863-889 (2016)

9. F. Palsson, J. R. Sveinsson, M. O. Ulfarsson, J. A. Benediktsson, in 2015 IEEE International Geoscience and Remote Sensing Symposium (IGARSS), Model based pansharpening method based on TV and MTF deblurring (IEEE, 2015), pp. 33-36

10. W. Li, Y. Li, Q. Hu, L. Zhang, Model-based variational pansharpening method with fast generalized intensity-hue-saturation. J. Appl. Remote. Sens. 13(3), 036513 (2019)

11. T.-M. Tu, S.-C. Su, H.-C. Shyu, P. S. Huang, A new look at IHS-like image fusion methods. Inf. Fusion. 2(3), 177-186 (2001)

12. P. Kwarteng, A. Chavez, Extracting spectral contrast in Landsat Thematic Mapper image data using selective principal component analysis. Photogramm. Eng. Remote Sens. 55(1), 339-348 (1989)

13. B. Aiazzi, S. Baronti, M. Selva, Improving component substitution pansharpening through multivariate regression of ms + pan data. IEEE Trans. Geosci. Remote Sens. 45(10), 3230-3239 (2007)

14. A. R. Gillespie, A. B. Kahle, R. E. Walker, Color enhancement of highly correlated images. II. Channel ratio and "chromaticity" transformation techniques. Remote Sens. Environ. 22(3), 343-365 (1987)

15. J. Liu, Smoothing filter-based intensity modulation: a spectral preserve image fusion technique for improving spatial details. Int. J. Remote Sens. 21(18), 3461-3472 (2000)

16. B. Aiazzi, L. Alparone, S. Baronti, A. Garzelli, M. Selva, MTF-tailored multiscale fusion of high-resolution MS and Pan imagery. Photogramm. Eng. Remote Sens. 72(5), 591-596 (2006)

17. M. M. Khan, J. Chanussot, L. Condat, A. Montanvert, Indusion: fusion of multispectral and panchromatic images using the induction scaling technique. IEEE Geosci. Remote Sens. Lett. 5(1), 98-102 (2008)

18. K. He, J. Sun, X. Tang, Guided image filtering. IEEE Trans. Pattern. Anal. Mach. Intell. 35(6), 1397-1409 (2012)

19. Y. Yang, W. Wan, S. Huang, F. Yuan, S. Yang, Y. Que, Remote sensing image fusion based on adaptive IHS and multiscale guided filter. IEEE Access. 4, 4573-4582 (2016)

20. W. Shi, S. Liu, F. Jiang, D. Zhao, Z. Tian, Anchored neighborhood deep network for single-image super-resolution. EURASIP J. Image Video Process. 2018(1), 34 (2018)

21. G. Scarpa, S. Vitale, D. Cozzolino, Target-adaptive CNN-based pansharpening. IEEE Trans. Geosci. Remote Sens. 56(9), 5443-5457 (2018)

22. S. Huang, J. Wu, Y. Yang, P. Lin, Multi-frame image super-resolution reconstruction based on spatial information weighted fields of experts. Multidim. Syst. Sign. Process. 31(1), 1-20 (2020)

23. S. Baghersalimi, B. Bozorgtabar, P. Schmid-Saugeon, H. K. Ekenel, J.-P. Thiran, Dermonet: densely linked convolutional neural network for efficient skin lesion segmentation. EURASIP J. Image Video Process. 2019(1), 71 (2019)

24. A. Mehmood, M. Maqsood, M. Bashir, Y. Shuyuan, A deep Siamese convolution neural network for multi-class classification of Alzheimer disease. Brain Sci. 10(2), 84 (2020)

25. Y. Wang, H. Bai, L. Zhao, Y. Zhao, Cascaded reconstruction network for compressive image sensing. EURASIP J. Image Video Process. 2018(1), 77 (2018)

26. Y. Rao, L. He, J. Zhu, in 2017 International Workshop on Remote Sensing with Intelligent Processing (RSIP), A residual convolutional neural network for pan-shaprening (IEEE, 2017), pp. 1-4

27. W. Huang, L. Xiao, Z. Wei, H. Liu, S. Tang, A new pan-sharpening method with deep neural networks. IEEE Geosci. Remote Sens. Lett. 12(5), 1037-1041 (2015)

28. A. Azarang, H. E. Manoochehri, N. Kehtarnavaz, Convolutional autoencoder-based multispectral image fusion. IEEE Access. 7, 35673-35683 (2019)

29. S. Dolgikh, Spontaneous concept learning with deep autoencoder. Int. J. Comput. Intell. Syst. 12(1), 1-12 (2018)

30. W. CARPER, T. LILLESAND, R. KIEFER, The use of intensity-hue-saturation transformations for merging spot panchromatic and multispectral image data. Photogramm. Eng. Remote Sens. 56(4), 459-467 (1990)

31. S. Rahmani, M. Strait, D. Merkurjev, M. Moeller, T. Wittman, An adaptive IHS pan-sharpening method. IEEE Geosci. Remote Sens. Lett. 7(4), 746-750 (2010)

32. K. He, J. Sun, X. Tang, in European Conference on Computer Vision, Guided image filtering (Springer, 2010), pp. 1-14

33. C. N. Ochotorena, Y. Yamashita, Anisotropic guided filtering. IEEE Trans. Image Process. 29, 1397-1412 (2019)

34. Y. Bengio, I. Goodfellow, A. Courville, Deep Learning, vol. 1. (MIT Press, Massachusetts, USA, 2017)

35. Y. Song, W. Wu, Z. Liu, X. Yang, K. Liu, W. Lu, An adaptive pansharpening method by using weighted least squares filter. IEEE Geosci. Remote Sens. Lett. 13(1), 18-22 (2015)

36. A. Garzelli, F. Nencini, L. Capobianco, Optimal MMSE pan sharpening of very high resolution multispectral images. IEEE Trans. Geosci. Remote Sens. 46(1), 228-236 (2007) 
37. J. Choi, K. Yu, Y. Kim, A new adaptive component-substitution-based satellite image fusion by using partial replacement. IEEE Trans. Geosci. Remote Sens. 49(1), 295-309 (2010)

38. G. Masi, D. Cozzolino, L. Verdoliva, G. Scarpa, Pansharpening by convolutional neural networks. Remote Sens. 8(7), 594 (2016)

39. M. Imani, IEEE J. Sel. Top. Appl. Earth Obs. Remote Sens. 11(12), 4994-5004 (2018)

40. Z. Wang, A. C. Bovik, A universal image quality index. IEEE Signal Process. Lett. 9(3), 81-84 (2002)

41. P. Jagalingam, A. V. Hegde, A review of quality metrics for fused image. Aquat. Procedia. 4, 133-142 (2015)

42. P. Mhangara, W. Mapurisa, N. Mudau, Comparison of image fusion techniques using satellite pour I'Observation de la Terre (SPOT) 6 satellite imagery. Appl. Sci. 10(5), 1881 (2020)

43. G. P. Petropoulos, K. P. Vadrevu, C. Kalaitzidis, Spectral angle mapper and object-based classification combined with hyperspectral remote sensing imagery for obtaining land use/cover mapping in a Mediterranean region. Geocarto Int. 28(2), 114-129 (2013)

44. F. Palsson, J. R. Sveinsson, M. O. Ulfarsson, J. A. Benediktsson, Quantitative quality evaluation of pansharpened imagery: consistency versus synthesis. IEEE Trans. Geosci. Remote Sens. 54(3), 1247-1259 (2015)

45. L. Alparone, B. Aiazzi, S. Baronti, A. Garzelli, F. Nencini, M. Selva, Multispectral and panchromatic data fusion assessment without reference. Photogramm. Eng. Remote Sens. 74(2), 193-200 (2008)

46. T. Ranchin, B. Aiazzi, L. Alparone, S. Baronti, L. Wald, Image fusion-the arsis concept and some successful implementation schemes. ISPRS J. Photogramm. Remote. Sens. 58(1-2), 4-18 (2003)

\section{Publisher's Note}

Springer Nature remains neutral with regard to jurisdictional claims in published maps and institutional affiliations.

\section{Submit your manuscript to a SpringerOpen ${ }^{\odot}$ journal and benefit from:}

- Convenient online submission

- Rigorous peer review

- Open access: articles freely available online

- High visibility within the field

- Retaining the copyright to your article

Submit your next manuscript at $\gg$ springeropen.com 